\title{
Antimüllerian Hormone (AMH) Not Only a Marker for Prediction of Ovarian Reserve
}

\author{
R. HAMPL ${ }^{1}$, M. ŠNAJDEROVÁ ${ }^{2}$, T. MARDEŠI $\check{C ́}^{3}$ \\ ${ }^{1}$ Institute of Endocrinology, Prague, Czech Republic, ${ }^{2}$ Department of Pediatrics, Second Medical \\ School, Charles University and University Hospital Motol, Prague, Czech Republic, ${ }^{3}$ Sanatorium \\ Pronatal, Prague, Czech Republic
}

Received September 6, 2010

Accepted October 8, 2010

On-line November 29, 2010

\section{Summary}

The main characteristics of the Antimüllerian hormone from the points of view of biochemistry, molecular genetics, physiological functions and importance for diagnostics in reproductive endocrinology and related biomedical fields are reviewed. The role of the hormone in male and female development, its participation in oocyte maturation including selection of a dominant follicle are summarized, as well as its changes under various pathological situations in both sexes. The physiological changes of serum AMH leves in the life span in both sexes and their alterations under various pathological conditions are provided, too.

\section{Key words}

Antimüllerian hormone • Physiological function • Pathophysiology

- Male development • Reproduction

\section{Corresponding author}

R. Hampl, Institute of Endocrinology, Národní 8, 11694 Praha 1, Czech Republic. E-mail: rhampl@endo.cz

\section{Introduction}

The first mention about the antimüllerian hormone (AMH) is dated to 1940, when A. Jost pointed to a protein substance, formed in testes of mammals including man and different from testosterone, responsible for regression of Müllerian ducts. It was named "Müllerian Inhibiting Substance". It lasted almost
40 years before the protein was isolated and characterized, including the gene which coded for it. Soon after its receptors were described (La Marca and Volpe 2006). As we show below, AMH is formed also in women, and measurable levels occur in blood of both sexes. Among physicians it is mostly familiar as a predictor for successful in vitro fertilization, but its examination has a wider utilization.

\section{AMH-description}

Antimüllerian hormone is a peptide belonging to the family of TGF-beta growth factors, the unique effect of which in males is a regression of Müllerian ducts during embryonal development, leading to initiation of a further dvelopment towards the male phenotype. In males it is synthesized in Sertoli cells of the testes since the 5th week of the embryonal development and then during the whole life. It is also formed in females in ovaries from the 36 th week of gestation. During the female life until menopause it is exprimed in granulosa cells of small growing follicles (primary and preantral). On the other hand it is not formed in FSH-dependent (antral) follicles and also in atretic follicles. The hormone passes in the blood and its levels can be measured.

From the chemical point of view AMH is a peptide homodimer of molecular weight $140 \mathrm{kDa}$, consisting of two identical glycoprotein subunits, connected by disulfide bridges. The gene for human AMH is localized on the short arm chromosome 19 and is 
composed from 2750 nucletide bases. The fifth exon from the 3 'end codes for a biologically active part of the molecule, rich of glycine. Target organs for AMH in males are Müllerian ducts, and in both sexes gonads. The receptors for AMH are transmembrane heteromeric proteins, composed from two subunits, denoted Type I and II. As all the receptors for growth factors of the TGFbeta family, they do not use G-proteins and possess intrinsic kinase activity. Type II (better subunit) binds specifically the ligand leading thus to activation of the Type I, the intracellular part of which acts as threonin kinase. Activation of the latter starts a signal cascade resulting in a respective biological response. The gene encoding for AMH receptor is composed from 11 exons more than $8 \mathrm{kbp}$ in lenght and is localized on chromosome 12 (La Marca and Volpe 2006). Soon after mapping of the genes for AMH and its receptor, their mutations were also described (Picard and Belville 2002, di Clemente and Belville 2010).

\section{Physiology and pathophysiology of AMH in males}

The changes of AMH expression follow the development of the male reproductive system, first of all changes in the activity of hypothalamo-pituitary-gonadal (HPG) axis. They may be divided into four main stages: fetal and early postnatal period, childhood, puberty and adulthood (Grinspon and Rey 2010). Variations in AMH formation are well reflected by its blood levels.

\section{Fetal and early postnatal period}

As mentioned above, AMH is synthesized in Sertoli cells of fetal testes already in early stage of embryonal development. In this period hypothalamus already produces gonadoliberin, which stimulates secretion of pituitary gonadotropins - lutropin (LH) and follitropin (FSH). By action of LH on Leydig cells of fetal testes via present receptors relatively high amounts of testosterone are formed (as detected for instance in newborn's circulation). Testosterone is responsible for differentiation of Wolffian ducts. At the same time testosterone would inhibit AMH formation in Sertoli cells through androgen receptors (AR). Because, however, AR are still not expressed in appropriate amounts, the latter effect does not appear. On the other hand, FSH through its receptors on the membrane of Sertoli cells, stimulates AMH expression (Rey et al. 2009). FSH utilizes here the classical mechanism involving binding to the receptor and activation of adenylate cyclase effector through G-protein. Cyclic adenosine triphoshate (cAMP), formed by action of adenylate cyclase, activates a number of kinases, among the first proteinkinase A (PKA, one of the most common ones), thus starting a signalling cascade, leading to activation and following translocation of nuclear transcription factors. They bind then to the respective responsive elements (sequences of DNA specifically recognizing activated transforming factors, usualy consisting of 15 nucleotide bases) in the promotor region of the $\mathrm{AMH}$ gene, resulting in its expression. Inhibin B is also formed and Sertoli cells, constituting in this stage about one half of the whole testicular tissue, which undergo proliferation (Grinspon and Rey 2010). Serum levels of AMH are relatively high in this stage, comparable with the levels in childhood (roughly up to the eighth year of boys, as shown below) (Grinspon and Rey 2010).

\section{Childhood and early prepubertal period}

Childhood, until prepubertal stage is a period of a relative rest of the HPG axis and it is sometimes called a stage of "hypogonadotropic hypogonadism". Leydig cells produce only very low amounts of testosterone, almost a half of which in addition originates from adrenals. Sertoli cells are still immature and spermiogenesis is arrested in a premeiotic stage. The latter cells, however represent the main portion of testicular tissue and thanks to FSH stimulation produce AMH in amounts, comparable with prenatal period (Rey et al. 2009, Grinspon and Rey 2010).

\section{Puberty}

With the onset of puberty the secretion of hypothalamic gonadoliberin and both gonadotropins increase again, but the effect of LH is much more pronounced, due to inhibitory effect of inhibin B on FSH secretion. Leydig cells undergo further differentiation and dramatically increases testosterone formation, which invokes also maturation of Sertoli cells. The inhibitory effect of testosterone prevails over FSH stimulation, resulting in down-regulation of AMH expression, the levels of which rapidly sink. Germinal cells undergo meiosis and spermatogenesis begins. In the late puberty stage (Tanner V), the germinal cells, in contrast to Sertoli cells, represent already the major portion of testicular tissue. The secretion of AMH reaches adult values and it is maintained almost constant until the rest of life (Rey et al. 2009, Grinspon and Rey 2010). 


\section{Adulthood}

The situation described in the late puberty stage, as a result of stimulatory effect of FSH and prevailing inhibitory effect of testosterone on Sertoli cells is maintained in adulthood.

\section{AMH as a marker of male hypogonadism}

Apart from overt somatic disorders (malformation of external genitalia, gonadal dysgenesis, cryptorchism etc.), male hypogonadism, either central or primary, may be overlooked in the early as well as in the later childhood, if the diagnosis is based only on investigation of gonadotropins and testosterone. As mentioned already, Sertoli cells in this period constitute the main portion of testicular tissue and small testes are above all a consequence of their lack. Lower AMH levels than those at the corresponding age are thus a very good marker of congenital central hypogonadism. On the other hand, in boys not treated with recombinant FSH doesn't occur a characteristic AMH decline at the age of puberty. Low AMH levels are typical for precocious puberty due to inhibitory effect of testosterone, while persisting high AMH levels at the age of physiological puberty indicate its delay. Besides hypogonadism AMH may thus help to a more accurate diagnosis of disorders in onset and duration of puberty (Grinspon and Rey 2010).

\section{The role of $\mathrm{AMH}$ in females}

AMH may be detected in female subjects since perinatal stage until menopause. The levels of the hormone in blood are, in comparison with those found in males, by order of magnitude lower. AMH inhibits the initial selection of follicles for further developmental stages (the selection of preantral and small antral follicles) and thus participates at selection of the dominant follicle, the development and growth of which is dependent on FSH stimulation. Increasing number of infertile couples in developed countries, as well as increasing numbers of women at their advanced reproductive age trying to conceive evoke the need of test, enabling more accurate prediction of their ovarian function. Determination of AMH belongs to modern parameters, fulfilling enough these requirements (La Marca and Volpe 2006, Visser et al. 2006).

\section{AMH as a marker of ovarian reserve}

The levels of AMH reflect the number of preantral follicles and thus are a marker of oocyte pool - a germinal reserve of the ovary for reproduction. Plasma levels of AMH tightly correlated with a number of mature follicles (AFC $=$ antral follicle count), as assessed by transvaginal ultrasonography (correaltion coefficient 0.66-0.71) and also with AMH concentrations measured in a follicular fluid (Kaya et al. 2010). Determination of plasma AMH in women of fertile age enables thus to assess with a greater specificity and sensitivity the extent of ovarian reserve than determination of FSH together with steroid hormones and inhibin, among other because $\mathrm{AMH}$ acts first of all paracrine and is not involved in feed-back mechanisms of hypothalamo-pituitary-gonadal axis. That's also the reason that AMH levels are almost independent on the phase of the menstrual cycle, and usually a single mesurement is sufficient. It is advantageous for interpretation of the diagnosis and also from the economical point of view (Visser et al. 2006). As was demonstrated on a group of women with polycystic ovary syndrome (PCOS) who underwent in vitro fertilization (IVF), the effect of IVF on the base of $\mathrm{AMH}$ determination, as a percentage of successful pregnancy could be predicted with sensitivity 75.6 and specificity $77.3 \%$ (Visser et al. 2006).

\section{AMH as an early marker of ovarian function cessation}

Hand in hand with the decrease of the number of primary and preantral follicles with age, the production of AMH declines along with its blood levels. Low AMH levels and also their rapid decrease in certain time period are a marker of the drop off of an ovarian function (Visser et al. 2006). In women under hormonal anticonception the proper function of ovaries cannot be evaluated without interruption of hormone administration for a period of 2-3 cycles, what brings about difficulties in a daily practice. Determination of FSH, LH and steroid hormone levels need not provide, in contrast to $\mathrm{AMH}$, a reliable information because of certain residual gonadal activity.

\section{AMH and PCOS}

Besides its utilization in IVF, determination of $\mathrm{AMH}$ may serve as an additional marker in the diagnostics of polycystic ovary syndrome, where increased AMH levels reflect the severity of the disease. Positive correlation of serum AMH with the number of antral follicles was found also in patients with PCOS. An increased production of $\mathrm{AMH}$ induces a decrease of sensitivity to FSH at receptor level, necessary for the growth of follicles. It leads to an increase of the number 
of antral follicles on the detriment of their size: the number of small antral follicles $2-5 \mathrm{~mm}$ in size increases, restraining thus selection of the dominant follicle. Such a situation is clinically characterized by anovulation cycles, manifesting themselve as oligo- or amenorrhea (Visser et al. 2006). In addition AMH inhibits peripheral aromatase, leading to hyperandrogenemia, another typical feature of PCOS. As mentioned already, secretion of AMH and its serum levels decrease with age. In patients with PCOS this decline was surprisingly less distinct, indicating a slowed-down ovarian ageing, which may be explained by suppression of growth and differentiation of primordial follicles by high levels of AMH (Visser et al. 2006, Kaya et al. 2010, Pellat et al. 2010, Park et al. 2010a).

When investigating possible genetical causes of PCOS, the question arose whether described mutations of AMH encoding gene are related to the disease. A genotypic analysis, however, did not reveal association of AMH gene mutations with PCOS, though they may contribute to aggravation of some features of the disease (Kevenaar et al. 2008, Sproul et al. 2010).

Besides hyperandrogenemia caused by increase of free testosterone, a characterisitic attribute of PCOS is insulinoresistance, usually expressed as the HOMA index, replacing in current medical practice euglycemic clamp. The studies of association of AMH levels with insulinoresistance surprisingly did not prove an unequivocal correlation among the investigated parameters: whilst in non-obese PCOS pacients the HOMA values as expected correlated positively with AMH levels (Nardo et al. 2009), another study including also obese women (Chen et al. 2008) reported contradictory results: AMH correlated negatively with HOMA-index and also with BMI. It points to importance of obesity as one of the factors tightly bound with AMH regulation. A negative correlation between $\mathrm{AMH}$ and HOMA has been also found in healthy women without PCOS (Park et al. 2010b). In this connection we should emphasize importance of sex hormone-binding (SHBG) determination, as an excellent laboratory marker for PCOS, thanks to its involvement in endocrine regulations, first of all in gonadal and insular axes. It is well known that androgens suppress hepatal SHBG production, while estrogens increase it. Another SHBG feature is its negative association with insulinemia. A result of lowered SHBG levels is an increase of free androgens, which invoke further decrese of SHBG (Hampl and Stárka 1996). SHBG is thus one of the connecting links in PCOS pathophysiology and obesity and it would be interesting to follow up relation of this protein to AMH.

\section{AMH as a tumor marker}

Serum AMH is a good marker of tumors originating from granulosa cells. Along with inhibin its determination was successfully tested as a marker of early diagnosis and response to the treatment. AMH appeared to be more specific, while sensitivity of both hormones was comparable (La Marca and Volpe 2007, Geerts et al. 2009). The values of AMH in these patients correlated well with the size of the tumor (Chang et al. 2009). AMH may be used also for the follow up of gonadal function in reproduction in subjects who underwent therapy possessing undesired side effects on gonads (oncological therapy, immunosuppression). As an example we may bring in the study on females, who underwent in childhood a chemotherapy for Hodgkin lymphoma, in which lowered AMH levels reflected well a decreased ovarian reserve (van Beek et al. 2007). Recent research brought evidence that $\mathrm{AMH}$ determination may serve as a tool for diagnosis of some other neoplasia, as for instance a prostate cancer and could be used for detection of tumor recurrence The results however were not definite (Eldar-Geva et al. 2010).

\section{The levels of AMH in serum and the methods for its determination}

Serum AMH levels are in the literature usually given in mass units $(\mathrm{ng} / \mathrm{ml}$ or $\mu \mathrm{g} / \mathrm{l})$, which are used also in this article, though according to a good laboratory practice, more accurate is usage of S.I. units (pmol/l). The respective converting factor is $[\mathrm{pmol} / \mathrm{l}]=7.14 \mathrm{x}[\mathrm{ng} / \mathrm{ml}]$. In the Table 1 the medians and standard deviations of physiological levels are provided for women in a fertile age and in men and boys as reported in the literature. Out of many reports we show the extract of the data from representative reviews from 2004-2009 (Laven et al. 2004, Fréour et al. 2007, Knauff et al. 2009, La Marca et al. 2009). In the recent review of La Marca et al. (2009) the survey of AMH levels in PCOS patients is given from eight authors published since 2002 to 2007. The data in Table 1 are medians of these values. 
Table 1. Physilogical levels of AMH (in $\mathrm{ng} / \mathrm{ml}$ ) in females and males according to age and in women with PCOS.

\begin{tabular}{|c|c|c|c|c|c|}
\hline \multirow{2}{*}{ Age/puberty stage/ } & \multicolumn{2}{|c|}{ Women and girls } & \multicolumn{2}{|c|}{ Men and boys } & \multirow{2}{*}{ Literature } \\
\hline & Median & S.D. & Median & S.D. & \\
\hline Until the $1^{\text {st }}$ month & 1.00 & 0.90 & 57.7 & 21.6 & Grinspon and Rey 2010 \\
\hline 1 month -4.9 years & 2.85 & 2.80 & 104.0 & 32.6 & Grinspon and Rey 2010 \\
\hline $5-12$ years & 3.20 & 2.70 & & & Grinspon and Rey 2010 \\
\hline Tanner I (boys) & & & 72.3 & 38.3 & Grinspon and Rey 2010 \\
\hline Tanner II (boys) & & & 34.9 & 17.6 & Grinspon and Rey 2010 \\
\hline Tanner III (boys) & & & 13.7 & 9.1 & Grinspon and Rey 2010 \\
\hline Tanner IV and $V$ and adults (both sexes) & 1.90 & 2.60 & 5.9 & 5.3 & Grinspon and Rey 2010 \\
\hline Women with PCOS & 6.90 & 1.05 & & & La Marca et al. 2009 \\
\hline
\end{tabular}

Given are medians and standard deviations from the means.

As mentioned already, AMH levels in healthy women decline continually with age reaching nonmeasurable values after menopause. They are higher in women with PCOS and, on the other hand, very low in women with an ovarian failure. More detailed data on $\mathrm{AMH}$ according to the age and at various pathological situations in females may be found in the cited reviews (Laven et al. 2004, Knauff et al. 2009, La Marca et al. 2009), in boys and men in (Rey et al. 2009, Grinspon and Rey 2010).

Since the end of nighties the immunological kits are available for $\mathrm{AMH}$ determination in body fluids (serum, plasma and also a follicular fluid). They usually apply sandwich systems with two specific antibodies to AMH: the first bound to a solid phase (in most instances the tube walls or titration wells), and the second labeled with biotin, to which the streptavidin-labeled enzyme is bound (usually horse-radish peroxidase). After addition of the substrate (a chromogenic conjugate which affords a a coloured product after cleavage by the enzyme) its absorbance is measured. The inserted streptavidin-biotin system increases the specificity and sensitivity of the method, which amounts approximately $1 \mathrm{ng} / \mathrm{ml}$.

\section{Conclusion}

In advanced countries the determination of $\mathrm{AMH}$ belongs to a standard constituent of laboratory examination of the above mentioned states. The aim of this minireview is to inform the clinicians and scientists about the wide possibilities of utilization of $\mathrm{AMH}$ determination. Last but not least an economical advantage of AMH determination over more expensive investigations used at IVF should be mentioned here, and also its contribution to early and targeted diagnosis of a number of disorders. One of the reason of this paper is to support the listing of $\mathrm{AMH}$ determination to the laboratory tests reimbursed by health insurance companies.

\section{Conflict of Interest}

There is no conflict of interest.

\section{Acknowledgement}

This article is dedicatied to the $80^{\text {th }}$ birthday of Professor Luboslav Stárka, MD, DSc.

\section{References}

CHANG HL, PAHLAVAN N, HALPERN EF, MACLAUGHLIN DT: Serum Müllerian Inhibiting Substance/antiMüllerian hormone levels in patients with adult granulosa cell tumors directly correlate with aggregate tumor mass as determined by pathology or radiology. Gynecol Oncol 114: 57-60, 2009.

CHEN MJ, YANG WS, CHEN CL, WU MY, YANG YS, HO HN: The relationship between anti-Mullerian hormone, androgen and insulin resistance on the number of antral follicles in women with polycystic ovary syndrome. Hum Reprod 23: 952-957, 2008. 
Di CLEMENTE N, BELVILLE C: Anti-Müllerian hormone receptor defect. Best Pract Res Clin Endocrinol Metab 20: 599-610, 2006.

ELDAR-GEVA T, LIBERTY G, CHERTIN B, FRIDMANS A, FARKAS A, MARGALIOTH EJ, SPITZ IM: Relationships between FSH, inhibin B, anti-Mullerian hormone, and testosterone during long-term treatment with the GnRH-agonist histrelin in patients with prostate cancer. Eur J Endocrinol 162: 177-181, 2010.

FRÉOUR T, MIRALLIÉ S, BACH-NGOHOU K, DENIS M, BARRIĖRE P, MASSON D: Measurement of serum antiMüllerian hormone by Beckman Coulter ELISA and DSL ELISA: comparison and relevance in assisted reproduction technology (ART). Clin Chim Acta 375: 162-164, 2007.

GEERTS I, VERGOTE I, NEVEN P, BILLEN J: The role of inhibins B and antimüllerian hormone for diagnosis and follow-up of granulosa cell tumors. Int J Gynecol Cancer 19: 847-855, 2009.

GRINSPON RP, REY RA: Anti-müllerian hormone and Sertoli cell function in paediatric male hypogonadism. Horm Res Paediatr 73: 81-92, 2010.

HAMPL R, STÁRKA L: Sex hormone-binding globulin in endocrine regulation (minireview). Endocr Regul 30: 57-65, 1996.

KAYA C, PABUCCU R, SATIROGLU H: Serum antimüllerian hormone concentrations on day 3 of the in vitro fertilization stimulation cycle are predictive of the fertilization, implantation, and pregnancy in polycystic ovary syndrome patients undergoing assisted reproduction. Fertil Steril 94: 2202-2207, 2010.

KEVENAAR ME, LAVEN JS, FONG SL, UITTERLINDEN AG, DE JONG FH, THEMMEN AP, VISSER JA: A functional anti-mullerian hormone gene polymorphism is associated with follicle number and androgen levels in polycystic ovary syndrome patients. J Clin Endocrinol Metab 93: 1310-1306, 2008.

KNAUFF EA, EIJKEMANS MJ, LAMBALK CB, TEN KATE-BOOIJ MJ, HOEK A, BEERENDONK CC, LAVEN JS, GOVERDE AJ, BROEKMANS FJ, THEMMEN AP, DE JONG FH, FAUSER BC; DUTCH PREMATURE OVARIAN FAILURE CONSORTIUM: Anti-Mullerian hormone, inhibin B, and antral follicle count in young women with ovarian failure. J Clin Endocrinol Metab 94: 786-792, 2009.

LA MARCA A, BROEKMANS FJ, VOLPE A, FAUSER BC, MACKLON NS; ESHRE SPECIAL INTEREST GROUP FOR REPRODUCTIVE ENDOCRINOLOGY-AMH ROUND TABLE: Anti-Mullerian hormone (AMH): what do we still need to know? Hum Reprod 24: 2264-2275, 2009.

LA MARCA A, VOLPE A: Anti-Müllerian hormone (AMH) in female reproduction: is measurement of circulating AMH a useful tool? Clin Endocrinol (Oxf) 64: 603-610, 2006.

LA MARCA A, VOLPE A: The Anti-Mullerian hormone and ovarian cancer. Hum Reprod Update 13: 265-273, 2007.

LAVEN JS, MULDERS AG, VISSER JA, THEMMEN AP, DE JONG FH, FAUSER BC: Anti-Müllerian hormone serum concentrations in normoovulatory and anovulatory women of reproductive age. $J$ Clin Endocrinol Metab 89: 318-323, 2004.

NARDO LG, YATES AP, ROBERTS SA, PEMBERTON P, LAING I: The relationships between AMH, androgens, insulin resistance and basal ovarian follicular status in non-obese subfertile women with and without polycystic ovary syndrome. Hum Reprod 24: 2917-2923, 2009.

PARK AS, LAWSON MA, CHUAN SS, OBERFIELD SE, HOEGER KM, WITCHEL SF, CHANG RJ: Serum antimullerian hormone concentrations are elevated in oligomenorrheic girls without evidence of hyperandrogenism. J Clin Endocrinol Metab 95: 1786-1792, 2010.

PARK HT, CHO GJ, AHN KH, SHIN JH, KIM YT, HUR JY, KIM SH, LEE KW, KIM T: Association of insulin resistance with anti-Mullerian hormone levels in women without polycystic ovary syndrome (PCOS). Clin Endocrinol (Oxf) 72: 26-31, 2010.

PELLATT L, RICE S, MASON H: Anti-Mullerian hormone and polycystic ovary syndrome: A mountain too high? Reproduction 139: 825-833, 2010.

PICARD JY, BELVILLE C: Genetics and molecular pathology of anti-Mullerian hormone and its receptor. J Soc Biol 196: 217-221, 2002.

REY RA, MUSSE M, VENARA M, CHEMES HE: Ontogeny of the androgen receptor expression in the fetal and postnatal testis: its relevance on Sertoli cell maturation and the onset of adult spermatogenesis. Microsc Res Tech 72: 787-795, 2009. 
SPROUL K, JONES MR, MATHUR R, AZZIZ R, GOODARZI MO: Association study of four key folliculogenesis genes in polycystic ovary syndrome. BJOG 117: 756-760, 2010.

VAN BEEK RD, VAN DEN HEUVEL-EIBRINK MM, LAVEN JS, DE JONG FH, THEMMEN AP, HAKVOORTCAMMEL FG, VAN DEN BOS C, VAN DEN BERG H, PIETERS R, DE MUINCK KEIZER-SCHRAMA SM: Anti-Mullerian hormone is a sensitive serum marker for gonadal function in women treated for Hodgkin's lymphoma during childhood. J Clin Endocrinol Metab 92: 3869-3874, 2007.

VISSER JA, DE JONG FH, LAVEN JS, THEMMEN AP: Anti-Müllerian hormone: a new marker for ovarian function. Reproduction 131: 1-9, 2006. 